\title{
Expected loss bounds for authentication in constrained channels
}

\author{
Christos Dimitrakakis \\ EPFL \\ Lausanne, Switzerland \\ christos.dimitrakakis@epfl.ch
}

\author{
Aikaterini Mitrokotsa \\ EPFL \\ Lausanne, Switzerland \\ katerina.mitrokotsa@epfl.ch
}

\author{
Serge Vaudenay \\ EPFL \\ Lausanne, Switzerland \\ serge.vaudenay@epfl.ch
}

\begin{abstract}
We derive bounds on the expected loss for authentication protocols in channels which are constrained due to noisy conditions and communication costs. This is motivated by a number of authentication protocols, where at least some part of the authentication is performed during a phase, lasting $n$ rounds, with no error correction. This requires assigning an acceptable threshold for the number of detected errors and taking into account the cost of incorrect authentication and of communication. This paper describes a framework enabling an expected loss analysis for all the protocols in this family. Computationally simple methods to obtain nearly optimal values for the threshold, as well as for the number of rounds are suggested and upper bounds on the expected loss, holding uniformly, are given. These bounds are tight, as shown by a matching lower bound. Finally, a method to adaptively select both the number of rounds and the threshold is proposed for a certain class of protocols.
\end{abstract}

\section{INTRODUCTION}

Traditionally [1], [2], authentication is assumed to be taking place on an error-free channel, and error analysis is performed separately from cryptographic analysis of protocols. However, a number of authentication protocols have been proposed [3][11], where at least some part of the authentication is performed during a challenge-response phase lasting $n$ rounds with no error correction, due to a need to detect relay attacks by timing delays. The noise necessitates the acceptance of some responses which are not perfectly correct. In addition, increasing $n$ carries a significant cost. The problem is to strike an optimal balance between the increased number of rounds, the probability of falsely authenticating an illegitimate party, and failing to authenticate a legitimate party.

For example the rapid-bit exchange phase in an RFID distance-bounding protocol (i.e. [9]), satisfies these criteria. The phase lasts $n$ rounds, where an equal number of challenges and responses are sent. Since there is no error correction during that phase, some responses of a legitimate user may be erroneous. This necessitates the use of a tolerance threshold $\tau$, such that if the number of erroneous responses is lower than $\tau$, the communicating party is nevertheless authenticated. In addition, while increasing the number of rounds decreases the probability of incorrect authentication, it also increases communication costs. The problem then is how to select $n, \tau$ in an optimal manner.
This paper ${ }^{1}$ performs an expected loss analysis of the authentication problem. This is necessary, because of the nontrivial cost of increasing the number of rounds $n$, the lack of an error-free channel and the need to trade off optimally the costs of incorrectly authenticating an illegitimate entity, or failing to authenticate a legitimate one.

We place the problem in a decision-theoretic framework. We assign a loss $\ell_{A}$ to the event that we authenticate a malicious party $A$-which we call the attacker - a loss $\ell_{U}$ to the event that we fail to authenticate a valid party $U$ - which we call the user - and a loss $\ell_{B}$ for each round of the challenge-response phase, such that the total communication cost is $n \ell_{B}$. Adding a non-negligible cost to the communications is of fundamental importance in resource-constrained environments. Otherwise, $n$ can be made as large as necessary to make the probability of authentication mistakes infinitesimal. Our goal is to select $n$ and $\tau$ so as to minimise the expected loss $\mathbb{E} L$ of the authentication system. This is achieved through a finite sample analysis.

The paper is organised as follows. Section II introduces notation and our framework. Section III contains the expected loss analysis under noise. In particular, Sec. III-A suggests a method to calculate the threshold accompanied by finite sample upper loss bounds and a matching lower bound, while Sec. III-B provides a further loss bound by selecting an near-optimal number of rounds $n$. These results only require two reasonable assumptions: that the expected error of the attacker is higher than that of the user and that the errors are independent in each round, something that can be achieved by appropriate protocol design. Section IV applies the above analysis to a number of currently used protocols. Section V suggests a high-probability method for estimating the channel noise and presents the results of simulation experiments that compare our choice of threshold with other approaches, based on thresholds derived using asymptotic approximations. Finally, Sec. VI concludes the paper with a discussion of related work. For completeness, the appendix provides some useful auxiliary results regarding the finite sample and the asymptotic derivations.

\footnotetext{
${ }^{1}$ Preliminary versions of this paper were published as arXiv:1009.0278 [12].
} 


\section{Preliminaries}

We consider sequences $x=x_{1}, \ldots, x_{n}$ with all $x_{i}$ in some alphabet $\mathcal{X}$ and $x \in \mathcal{X}^{n}$. We write $\mathcal{X}^{*} \triangleq \bigcup_{n=0}^{\infty} \mathcal{X}^{n}$ for the set of all sequences. We use $\triangleq$ to indicate a definition. $\mathbb{P}(A)$ denotes the probability of event $A$, while $\mathbb{E}$ denotes expectations so that $\mathbb{E}(X \mid A)=\sum_{u \in \Omega} u \mathbb{P}(X=u \mid A)$ denotes the conditional expectation of a random variable $X \in \Omega$ when $A$ is true. Finally, $\mathbb{I}\{A\}$ is an indicator function equal to 1 when $A$ is true and 0 otherwise.

We consider additive-error challenge-response authentication protocols. In such protocols, a verifier $\mathcal{V}$ grants access to a prover $\mathcal{P}$, if the latter can demonstrate its identity via possession of a shared secret. The protocol has three phases: (i) An initialisation phase. (ii) A challenge-response phase, lasting $n$ rounds, performed without error correction under noisy conditions. (iii) A termination phase. During the challenge-response phase the verifier $\mathcal{V}$ sends $n$ challenges $c_{1}, \ldots, c_{n}$, with $c_{k} \in \mathcal{X}$, to the prover $\mathcal{P}$, which responds by transmitting $n$ responses $r_{1}, \ldots, r_{n}$, with $r_{k} \in \mathcal{X}$. We use $c=\left(c_{k}\right)_{k=1}^{n}$, and $r=\left(r_{k}\right)_{k=1}^{n}$ to denote the complete challenge and response sequences respectively. The verifier $\mathcal{V}$ uses an error function $\mathcal{E}: \mathcal{X} \times \mathcal{X} \rightarrow[0,1]$ to calculate an error $\varepsilon_{i}=\mathcal{E}\left(r_{i}, c_{i}\right)$ for the $i$-th round. The errors when interacting with a valid user may be non-zero due to noise constraints in the channel. However, as the attacker has to resort to guessing the responses, the expected error of the attacker should be higher than that of the user.

In order to trade off false acceptances with false rejections, we use a threshold value $\tau$, such that a prover is accepted if and only if the total error observed is smaller than $\tau$. The verifier $\mathcal{V}$ calculates the total error $\varepsilon \triangleq \sum_{i=1}^{n} \varepsilon_{i}$, and rejects the prover $\mathcal{P}$, if and only if $\varepsilon \geq \tau$.

The relation of $\varepsilon$ to the challenge and response strings $c$ and $r$ strongly depends on the protocol. In order to make our analysis generally applicable, we make the following assumption about the protocol.

Assumption 1. We assume there exists some $p_{A} \leq \mathbb{E}\left(\varepsilon_{i} \mid A\right)$, a lower bound on the expected per-round error of the attacker and some $p_{U} \geq \mathbb{E}\left(\varepsilon_{i} \mid U\right)$, an upper bound on the error of a legitimate user. In addition, we assume that the protocol is such that all errors are independently (but not necessarily identically) distributed.

These bounds depend on the noise during the challengeresponse phase and on the protocol under consideration. We shall return to them in section IV.

\section{EXPECTED LOSS ANALYSIS}

We now specify our potential losses. For every round of the challenge-response phase, we suffer loss $\ell_{B}$, due to the cost in time and energy of transmission. In addition, we suffer a loss of $\ell_{A}$ for each false acceptance and a loss $\ell_{U}$ for each false rejection. ${ }^{2}$ Given that we perform $n$ rounds, the total

\footnotetext{
${ }^{2}$ These losses are subjectively set to application-dependent values. Clearly, for cases where falsely authenticating an attacker the impact is severe, $\ell_{A}$ must be much greater than $\ell_{U}$.
}

loss when the prover $\mathcal{P}$ is either the legitimate user $U$ or the attacker $A$ is given by:

$$
L= \begin{cases}n \ell_{B}+\ell_{U}, & \text { if } \varepsilon \geq \tau \text { and } \mathcal{P}=U \\ n \ell_{B}+\ell_{A}, & \text { if } \varepsilon<\tau \text { and } \mathcal{P}=A \\ n \ell_{B}, & \text { otherwise. }\end{cases}
$$

Armed with this information, we can now embark upon an expected loss analysis. We wish to devise an algorithm that guarantees an upper bound on the expected loss $\mathbb{E} L$ of the authentication system. To start with, we note that the expected loss when the communicating party is an attacker $A$ or the user $U$, is given respectively by:

$$
\begin{aligned}
& \mathbb{E}(L \mid A)=n \ell_{B}+\mathbb{P}(\varepsilon<\tau \mid A) \cdot \ell_{A}+\mathbb{P}(\varepsilon \geq \tau \mid A) \cdot 0 \\
& \mathbb{E}(L \mid U)=n \ell_{B}+\mathbb{P}(\varepsilon<\tau \mid U) \cdot 0+\mathbb{P}(\varepsilon \geq \tau \mid U) \cdot \ell_{U}
\end{aligned}
$$

The expected loss is in either case bounded by the worst-case expected loss:

$$
\mathbb{L} \triangleq \max \{\mathbb{E}(L \mid A), \mathbb{E}(L \mid U)\} \geq \mathbb{E} L
$$

If we can find an expression that bounds both $\mathbb{E}(L \mid A)$ and $\mathbb{E}(L \mid U)$, we automatically obtain a bound on the expected loss, $\mathbb{E} L$.

\section{A. Choice of threshold}

We want a threshold $\tau$ such that no matter whether the prover $\mathcal{P}$ is the attacker $A$ or the legitimate user $U$ the expected loss $\mathbb{E}(L \mid \mathcal{P})$ is as small as possible. As we increase the threshold $\tau, \mathbb{E}(L \mid \mathcal{P}=U)$ decreases, while $\mathbb{E}(L \mid \mathcal{P}=A)$ increases and vice-versa. Intuitively, to minimise the worstcase expected loss, we can use $\tau$ such that $\mathbb{E}(L \mid \mathcal{P}=A, \tau)=$ $\mathbb{E}(L \mid \mathcal{P}=U, \tau)$. A threshold $\tau$ minimising an upper bound on the worst-case expected loss is given in Theorem 1. As an intermediate step, we obtain a bound on the worst-case expected loss for any given threshold $\tau$. Formally, we can show the following:

Lemma 1. Let $\varepsilon_{i} \in[0,1]$ be the error of the $i$-th round. If, for all $i>0$, it holds that $\mathbb{E}\left(\varepsilon_{i} \mid A\right) \geq p_{A}$ and $\mathbb{E}\left(\varepsilon_{i} \mid U\right) \leq p_{U}$, for some $p_{A}, p_{U} \in[0,1]$ such that $n p_{U} \leq \tau \leq n p_{A}$, then:

$$
\begin{aligned}
\mathcal{L}(n ; \tau) & \triangleq n \ell_{B}+\max \left\{e^{-\frac{2}{n}\left(n p_{U}-\tau\right)^{2}} \ell_{U}, e^{-\frac{2}{n}\left(n p_{A}-\tau\right)^{2}} \ell_{A}\right\} \\
& \geq \max \{\mathbb{E}(L \mid A), \mathbb{E}(L \mid U)\} \geq \mathbb{E} L
\end{aligned}
$$

Proof: The expected loss when $\mathcal{P}=A$, is simply:

$$
\begin{aligned}
\mathbb{E}(L \mid A) & =n \ell_{B}+\mathbb{P}\left(\sum_{i} \varepsilon_{i}<\tau \mid A\right) \ell_{A} \\
& \leq n \ell_{B}+\mathbb{P}\left(\sum_{i} \varepsilon_{i}-n \mathbb{E}\left(\varepsilon_{i} \mid A\right)<\tau-n p_{A} \mid A\right) \ell_{A} \\
& \leq n \ell_{B}+e^{-\frac{2}{n}\left(n p_{A}-\tau\right)^{2}} \ell_{A},
\end{aligned}
$$

the last two steps used the fact that $\mathbb{E}\left(\varepsilon_{i} \mid A\right) \geq p_{A}$ and the Hoeffding inequality (18). Specifically, in our case, Lemma 3 (page 7) applies with $X_{i}=\epsilon_{i}$. Then, it is easy to see that 
$\mu_{i}=\mathbb{E}\left(\varepsilon_{i} \mid A\right)$ for all $i$ and $b_{i}-a_{i}=1$, so $\mathbb{P}\left(\epsilon<n p_{A}+\right.$ $n t \mid A) \leq e^{-2 n t^{2}}$. By setting $\tau=n p_{A}+n t$, we obtain $t=\left(\tau-n p_{A}\right) / n$, which we can plug into the above inequality, thus arriving at the required result.

The other case, $\mathcal{P}=U$, is handled similarly and we conclude that $\mathbb{E}(L \mid U) \leq n \ell_{B}+e^{-\frac{2}{n}\left(n p_{U}-\tau\right)^{2}} \ell_{U}$.

The given upper bound is tight, as can be seen by the following lemma, which proves a matching lower bound for the example of Bernoulli-distributed errors.

Lemma 2. Modifying our assumptions slightly, let $\varepsilon_{i} \in\{0,1\}$ be the Bernoulli-distributed error of the $i$-th round, such that, for all $i>0$, it holds that $\mathbb{P}\left(\varepsilon_{i} \mid A\right)=p_{A}$ and $\mathbb{P}\left(\varepsilon_{i} \mid U\right)=p_{U}$, for some $p_{A}, p_{U} \in[0,1]$ such that $n p_{U} \leq \tau \leq n p_{A}$, then:

$$
\begin{aligned}
\mathbb{E} L & \geq \min \{\mathbb{E}(L \mid A), \mathbb{E}(L \mid U)\} \\
& \geq n \ell_{B}+\left(\frac{n}{\tau}\right)^{\tau} \min \left\{\ell_{A} p_{A}^{\tau}\left(1-p_{A}\right)^{n-\tau}, \ell_{U} p_{U}^{\tau}\left(1-p_{U}\right)^{n-\tau}\right.
\end{aligned}
$$

Proof: The expected loss when $\mathcal{P}=U$, is simply:

$$
\begin{aligned}
\mathbb{E}(L \mid U) & =n \ell_{B}+\mathbb{P}\left(\sum_{i} \varepsilon_{i} \geq \tau \mid U\right) \ell_{U} \\
& \geq n \ell_{B}+\mathbb{P}\left(\sum_{i} \varepsilon_{i}=\tau \mid U\right) \ell_{U} \\
& =n \ell_{B}+\left(\begin{array}{l}
n \\
\tau
\end{array}\right) p_{U}^{\tau}\left(1-p_{U}\right)^{n-\tau} \ell_{U} \\
& \geq n \ell_{B}+\left(\frac{n p_{U}}{\tau}\right)^{\tau}\left(1-p_{U}\right)^{n-\tau} \ell_{U}
\end{aligned}
$$

The other case, $\mathcal{P}=A$, is handled similarly and we conclude that $\mathbb{E}(L \mid A) \geq n \ell_{B}+\left(\frac{n p_{A}}{\tau}\right)^{\tau}\left(1-p_{A}\right)^{n-\tau} \ell_{A}$

To see that the upper and lower bounds match, consider that $\mathcal{P}=U$ and $\tau=n c$ with $c<1$. Then Lemmas 1 and 2 give us:

$$
\begin{aligned}
n \ell_{B}+e^{-\frac{2}{n}\left(n p_{U}-\tau\right)^{2}} \ell_{U} & \geq n \ell_{B}+\left(\frac{n p_{U}}{\tau}\right)^{\tau}\left(1-p_{U}\right)^{n-\tau} \ell_{U} \\
e^{-\frac{2}{n}\left(n p_{U}-\tau\right)^{2}} & \geq\left(\frac{n p_{U}}{\tau}\right)^{\tau}\left(1-p_{U}\right)^{n-\tau} \\
e^{-2\left(p_{U}-c\right)^{2} n} & \geq e^{\left[c \ln \frac{p_{U}}{c}+(1-c) \ln \left(1-p_{U}\right)\right] n}
\end{aligned}
$$

Thus, the upper and lower bounds given for the expected loss $(\mathbb{E} L)$ are of the same order with regard to $n$.

Having bounded the loss suffered when choosing a specific threshold, we now choose a threshold $\hat{\tau}_{n}^{*}$ that minimises the above bound for fixed $n$. In fact, we can show that such a threshold results in a particular upper bound on the expected loss.

Theorem 1. Let $\rho \triangleq \ell_{A} / \ell_{U}$ and select

$$
\tau=\hat{\tau}_{n}^{*} \triangleq \frac{n\left(p_{A}+p_{U}\right)}{2}-\frac{\ln \rho}{4 \Delta}
$$

If $n p_{U} \leq \tau \leq n p_{A}$, then the expected loss $\mathbb{E} L$ is bounded by:

$$
\mathbb{E}\left(L \mid n, \hat{\tau}_{n}^{*}\right) \leq \mathcal{L}_{1}(n) \triangleq n \ell_{B}+e^{-\frac{n}{2} \Delta^{2}} \cdot \sqrt{\ell_{A} \ell_{U}} .
$$

with $\Delta \triangleq p_{A}-p_{U}$.
Proof: Substitute (7) in the first exponential of (5) to obtain:

$$
e^{-\frac{2}{n}\left(n p_{U}-\hat{\tau}^{*}\right)^{2}} \ell_{U}=e^{-\frac{n}{2} \Delta^{2}} e^{-\frac{\ln ^{2} \rho}{8 n \Delta^{2}}} \sqrt{\ell_{A} \ell_{U}} .
$$

It is easy to see that the exact same result is obtained by substituting (7) in the second exponential of (5). Thus, both $\mathbb{E}(L \mid A)$ and $\mathbb{E}(L \mid U)$ are bounded by the same quantity and consequently, so is $\max \{\mathbb{E}(L \mid A), \mathbb{E}(L \mid U)\}$. Thus,

$$
\mathcal{L}\left(n, \hat{\tau}_{n}^{*}\right) \leq n \ell_{B}+e^{-\frac{n}{2} \Delta^{2}} \sqrt{\ell_{A} \ell_{U}},
$$

where we simplified the bound by noting that $\frac{\ln ^{2} \rho}{8 n \Delta^{2}}>0$.

The intuition behind the algorithm and the analysis is that it is possible to bound the probability that $A$ makes less errors than expected, or that $U$ makes more than expected. For this reason, the $\hat{\tau}_{n}^{*}$ chosen in the theorem must lie between $n p_{U}$ gnd $n p_{A}$. This also implies a lower bound on the number of rounds $n$.

\section{B. Choice of the number of rounds}

Using similar techniques to those employed for obtaining a suitable value for the threshold, we now indicate a good choice for the number of rounds $n$ and provide a matching bound on the expected loss.

Theorem 2. Assume $\ell_{A}, \ell_{U}, \ell_{B}>0$. If we choose $\tau=\hat{\tau}_{n}^{*}$ and

$$
n=\hat{n}^{*} \triangleq \frac{\sqrt{1+2 C K}-1}{C},
$$

where $C=\Delta^{2}$ and $K=\sqrt{\ell_{A} \ell_{U}} / \ell_{B}$, then the expected loss $\mathbb{E} L$ is bounded by:

$$
\mathbb{E}\left(L \mid \hat{\tau}_{n}^{*}, \hat{n}^{*}\right) \leq \mathcal{L}_{2} \triangleq \sqrt{8 K / C} \cdot \ell_{B}=\frac{\sqrt{8 \ell_{B}}\left(\ell_{A} \ell_{U}\right)^{1 / 4}}{\Delta} .
$$

Proof: We shall bound each one of the summands of (8) by $\sqrt{2 K / C} \cdot \ell_{B}$. For the first term we have:

$$
\begin{aligned}
n \ell_{B} & =\frac{\sqrt{1+2 C K}-1}{C} \ell_{B} \leq \frac{\sqrt{1+2 C K}}{C} \ell_{B} \\
& \leq \frac{\sqrt{2 C K}}{C} \ell_{B}=\sqrt{\frac{2 K}{C}} \ell_{B} .
\end{aligned}
$$

For the second term, by noting that $e^{x} \geq 1+x$, we have:

$$
\begin{aligned}
\sqrt{\ell_{U} \ell_{A}} \cdot e^{-\frac{n}{2} \Delta^{2}} & \leq \frac{\sqrt{\ell_{U} \ell_{A}}}{1+\frac{n}{2} \Delta^{2}}=\frac{K \ell_{B}}{1+\frac{n C}{2}}=\frac{2 K \ell_{B}}{1+\sqrt{1+2 C K}} \\
& \leq \frac{2 K \ell_{B}}{\sqrt{1+2 C K}} \leq \frac{2 K \ell_{B}}{\sqrt{2 C K}}=\sqrt{\frac{2 K}{C} \ell_{B} .}
\end{aligned}
$$

Summing the two bounds, we obtain the required result.

This theorem proves that our worst-case expected loss $\mathbb{L}$ grows sublinearly both with increasing round cost (with rate $O\left(\epsilon^{1 / 2}\right)$ ) and with increasing authentication costs (with rate $\left.O\left(\epsilon^{1 / 4}\right)\right)$. Furthermore, the expected loss is bounded symmetrically for both user and attacker access. Finally, there is a strong dependence on the margin $\Delta$ between the attacker and the user error rates, which is an expected result. 


\section{ANALYSIS OF RFID THRESHOLDED PROTOCOLS}

Currently, the most well-known protocols employing an authentication phase without any error correction and under resource constraints are RFID distance bounding protocols. For that reason, we shall examine the properties of two such protocols, for which it is possible to derive expressions for $p_{A}, p_{U}$, given a symmetric channel noise.

More precisely, due to noise in the physical medium, in any exchange between $\mathcal{V}$ and $\mathcal{P}$, the former may send a symbol $x \in$ $\mathcal{X}$, while the latter may receive a symbol $\hat{x} \in \mathcal{X}$ such that $x \neq$ $\hat{x}$. We shall denote the probability of erroneous transmission in the data layer as: $\omega \triangleq \mathbb{P}(\hat{x}=j \mid x \neq j, x \neq \hat{x})$. For simplicity, we shall only treat the case of symmetric channel noise such that: $\mathbb{P}(\hat{x}=y \mid x \neq y)=\frac{1}{|\mathcal{X}|-1}, \forall y \neq x, x, y \in \mathcal{X}$.

The SwISS-KNIFE protocol [11] and the variant Hiтомi [7] are additive-error authentication protocols satisfying our assumptions. It is easy to show (for details see [7]) that, for those two protocols, under channel noise $\omega$, the expected error bounds $p_{A}, p_{U}$ are given by: $p_{A}=\frac{\omega+1}{2}, p_{U}=2 \omega$, where we note in passing that $p_{A} \geq p_{U}$ and so $\omega \leq \frac{1}{3}$. Finally, by substituting $\Delta=p_{A}-p_{U}=\frac{1-3 \omega}{2}$ in (8), we obtain the following bound for SWISS-KNIFE and HITOMI:

$$
\mathbb{E} L \leq n \ell_{B}+e^{-\frac{n(1-3 \omega)^{2}}{8}} \cdot \sqrt{\ell_{A} \ell_{U}}
$$

We have performed a number of experiments to test the efficacy of these protocols, when used in conjunction with our suggested, as well as the optimal values of the threshold and number of rounds. In all of the experiments shown here, we chose the following values for the losses: $\ell_{A}=10, \ell_{U}=1$, $\ell_{B}=10^{-2}$.

Figure 1(a) depicts the bound (11) on the expected loss, as well as the actual $\mathbb{E} L$ calculated via the binomial formula, when the threshold $\hat{\tau}_{n}^{*}$, calculated from (7), is used. We plot both the expected loss and the bound for two different channel noise levels $\omega \in\left\{10^{-1}, 10^{-2}\right\}$, where the number of rounds $n$ varies from 1 to 256 . Obviously, the bound is greater than the actual expected loss, while it approaches it exponentially fast as $n$ increases. In addition, the losses are higher when the amount of noise increases.

Furthermore, we can see that there are minimising values of $n$ for all cases. While they do not coincide for the bound and the actual expected loss, they are within a factor of two of each other. Finally, $\hat{n}^{*}$, the value of $n$ minimising the bound, is always greater than $n^{*} \triangleq \operatorname{argmin}_{n} \max _{\mathcal{P} \in A, U} \mathbb{E}(L \mid \mathcal{P}, n)$, the value of $n$ that minimises the worst-case expected loss. Since the probability of incorrect authentication always decreases with increasing $n$, this implies that any additional losses incurred by using $\hat{n}^{*}$ is due to transmission costs only.

Figure 1(b) examines the effect of noise in more detail. In particular, it depicts the worst-case expected loss for the optimal number of rounds $n^{*}$, denoted by $\mathbb{E}\left(L \mid n^{*}\right)$ in the legend. This is of course smaller than $\mathbb{E}\left(L \mid \hat{n}^{*}\right)$, the loss suffered by choosing $\hat{n}^{*}$, with the gap becoming smaller for larger error rates. Since when this occurs, the expected loss is

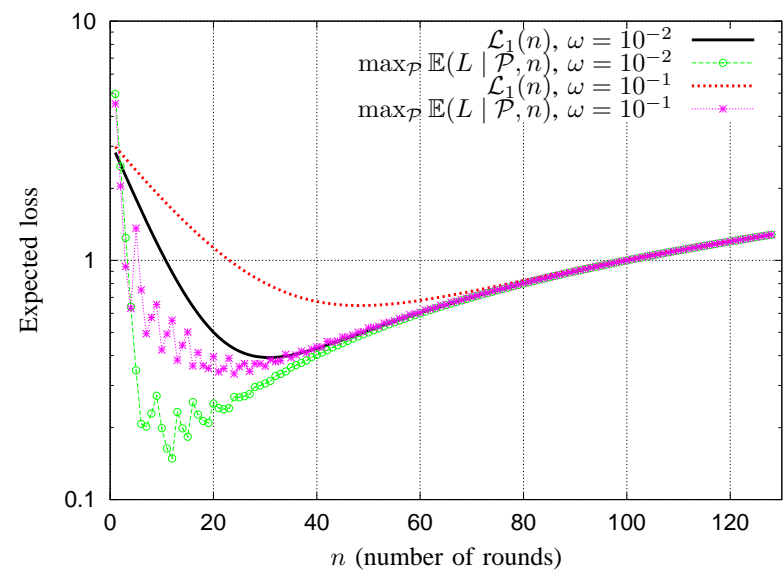

(a) The Expected Loss $\mathbb{E} L$ and the bound on the Expected Loss $\mathcal{L}_{1}$ vs. the number of bits $n$ exchanged during the rapid single bit exchange for various values of channel noise $\omega$.

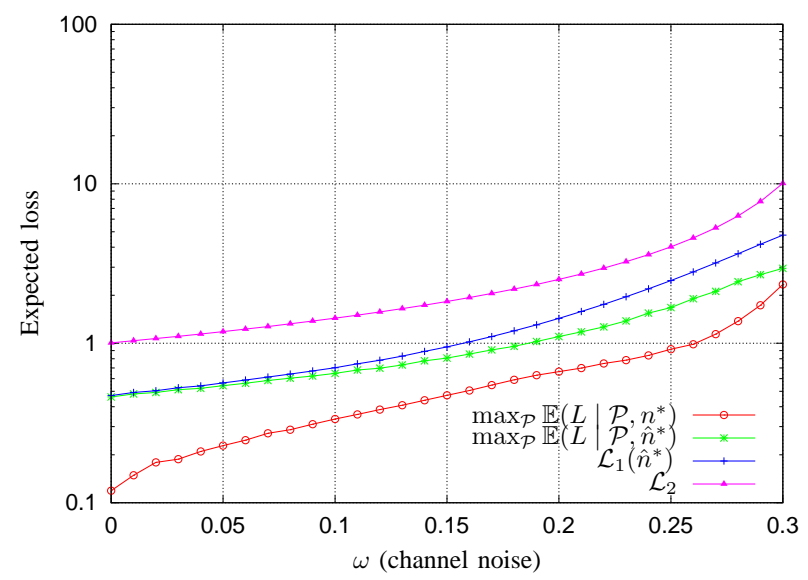

(b) The worst-case expected Loss $\mathbb{L}$ and the bounds $\mathcal{L}_{1}$ and $\mathcal{L}_{2}$ from theorems 1 and 2 respectively vs. the channel error rate $\omega$.

Fig. 1. Comparison of all losses.

very close to $\mathcal{L}_{1}$, this implies that the bound of the Theorem 1 can be further tightened for small $\omega$.

\section{Estimating $\omega$}

In this section, we discuss how it is possible to calculate the channel error rate $\omega$, which is used in the expressions for $p_{A}, p_{U}$. This can be done by leveraging the coding performed during the initial and final phases of the protocol. We assume some coding function $\Phi: \mathcal{X}^{m} \rightarrow \mathcal{X}^{k}$, with $k>m$, and a metric $\gamma$ on $\mathcal{X}^{k}$ (where usually $\mathcal{X}=\{0,1\}$ and $\gamma$ is the Hamming distance) such that:

$$
\gamma_{\min } \triangleq \min \left\{\gamma(\Phi(x), \Phi(y)): x, y \in \mathcal{X}^{m}, x \neq y\right\}
$$

is the minimum (Hamming) distance between valid codewords. For a given $x \in \mathcal{X}^{m}$, the source transmits $\phi=\Phi(x)$ and the sink receives $\hat{\phi}$, with $\phi, \hat{\phi} \in \mathcal{X}^{n}$. As before, we assume that the physical channel has a symmetric error rate $\omega=\mathbb{P}\left(\hat{\phi}_{i} \neq \phi_{i}\right)$, where $\phi_{i}$ denotes the $i$-th bit of $\phi$. This is then decoded as $\hat{x} \triangleq \operatorname{argmin}\left\{\gamma(\hat{\phi}, \Phi(y)): y \in \mathcal{X}^{m}\right\}$. Let $\theta$ be the number of 
errors in the string $\hat{\phi}$, or more precisely $\theta=\gamma(\phi, \hat{\phi})$. Let $\hat{\theta} \triangleq$ $\gamma(\Phi(\hat{x}), \hat{\phi})$ be the distance between the closest valid codeword $\Phi(\hat{x})$ and the received $\hat{\phi}$. If $\theta<\left(\gamma_{\min }-1\right) / 2$, then $\theta=\hat{\theta}$.

The crux of our method for estimating $\omega$ relies on the number of errors $\theta$ being less than $\left(\gamma_{\min }-1\right) / 2$, in which case, the estimated number of errors $\hat{\theta}$ will equal $\theta$. Let $\hat{\omega} \triangleq \frac{\hat{\theta}}{n}$ be our empirical error rate. In that case, the expected empirical error rate equals the true error rate. More formally:

$$
\mathbb{E}\left(\hat{\omega} \mid \theta \leq\left(\gamma_{\min }-1\right) / 2\right)=\omega .
$$

If $\theta>\left(\gamma_{\min }-1\right) / 2$ then the protocol fails in any case, due to decoding errors in the initial or final phases. If not, then the above equation holds and we can obtain high probability bounds for $\omega$ via the Hoeffding inequality (Appendix, Lemma $3)$. In particular, it is easy to show that, for any $\delta \in[0,1]$ :

$$
\mathbb{P}\left(|\hat{\omega}-\omega| \geq \sqrt{\frac{\ln 2 / \delta}{2 k}}\right) \leq \delta,
$$

by substituting the square-root term into (18), and setting $\mu_{i}=$ $\omega, \sum X_{i}=\hat{\theta}, a_{i}=0, b_{i}=1$. Consequently, for the SwISsKNIFE family of protocols the following values for $p_{A}$ and $p_{U}$ hold with probability $1-\delta$ :

$$
p_{A}=\frac{1+\hat{\omega}}{2}+\sqrt{\frac{\ln 2 / \delta}{8 k}}, \quad p_{U}=2 \hat{\omega}-\sqrt{\frac{2 \ln 2 / \delta}{k}} .
$$

Experimental investigations presented in the next section indicate that this choice has good performance in terms of expected loss.

\section{A. Evaluation Experiments}

We have performed some experiments to evaluate our methods in a more realistic setting, involving an RFID distance bounding protocol with a rapid-bit exchange phase. We perform simulations for two cases: Firstly, when a legitimate user $U$ is trying to get authenticated and secondly, when an adversary $A$ is trying to perform a mafia fraud attack [13]. We have estimated the worst-case expected loss by running $10^{4}$ experiments for each case, obtaining a pair of estimates $\hat{\mathbb{E}}(L \mid A), \hat{\mathbb{E}}(L \mid U)$ by averaging the loss $L$, as defined in (1), incurred in each experiment and taking the maximum of the two. In all of the experiments shown in this section, we chose the following values for the losses: $\ell_{A}=10, \ell_{U}=1$, $\ell_{B}=10^{-2}$, while we used $k=2^{10}$ for the coded messages in the initialisation phase.

The actual values $p_{A}, p_{U}$ depend on $\omega$, which is unknown. We compare three methods for choosing $p_{A}, p_{U}$. Firstly, guessing a value $\hat{\omega}$ for the channel noise. Secondly, using the maximum likelihood noise estimate $\hat{\omega}=\hat{\theta} / k$. In both cases, we simply use $\hat{\omega}$ as described at the beginning of Sec. IV to obtain $p_{A}, p_{U}$. In the third case, we use the high-probability bounds (15) for $p_{A}, p_{U}$, with an arbitrary value of $\delta$.

In the first experiment, we use the nearly-optimal threshold and number of rounds that we have derived in our analysis. In the second experiment, we replace our choice of threshold with a choice similar to that of Baignères et al. [14]. Their threshold is derived via a likelihood ratio test, which is asymptotically optimal (c.f. [15], [16]) but they do not consider specific losses. Since in our case we have unequal losses $\ell_{A}$ and $\ell_{U}$, we re-derive their threshold via a Bayesian test (to which a Bayesian formulation of the Neymann-Pearson lemma [15] applies) to obtain:

$$
\tilde{\tau}=\frac{n \ln \frac{1-p_{U}}{1-p_{A}}-\ln \rho}{\ln \frac{1-p_{U}}{1-p_{A}}-\ln \frac{p_{U}}{p_{A}} .}
$$

For equal losses, $\rho=1$, and $\tilde{\tau}$ equals the threshold used in [14]. Such tests have good asymptotic properties [15], [17]. Interestingly, for small $\Delta$, the form of $\tilde{\tau}$ is similar to $\hat{\tau}_{n}^{*}$ : Let $\bar{p}$ such that $p_{A}=\bar{p}+\Delta / 2$ and $p_{U}=\bar{p}-\Delta / 2$. Then (16) can be approximated by $\tau^{*}=n \bar{p}-\frac{\bar{p}(1-\bar{p})}{\Delta} \ln \rho$. More details on the derivation of (16) are given in Appendix B on page 7.

Figure 2 depicts the worst-case expected loss $\mathbb{L}$ as a function of the actual noise $\omega$. Figure 2(a) shows $\mathbb{L}$ using the threshold $\tau$ derived from our expected loss analysis (7), while in Figure 2(b) we use the asymptotically optimal threshold of (16). In both cases, we plot $\mathbb{L}$, while the actual noise $\omega$ is changing, for a number of different cases. Initially, we investigate the evolution of $\mathbb{L}$ for three arbitrarily chosen values $\hat{\omega} \in\left\{10^{-1}, 10^{-2}, 10^{-3}\right\}$. Additionally, we examine the evolution of the worst-case expected loss, when the noise is empirically estimated $\hat{\omega}=\hat{\theta} / n$ and finally when $p_{A}$ and $p_{U}$ are calculated via equation (15) with $\delta \in\left\{10^{-1}, 10^{-2}\right\}$.

As it can be seen in Figure 2, in all cases (using ours Figure 2(a) or Baignères et al. [14] threshold Figure 2(b)) the worst-case expected loss is very low for small values of the actual noise and increases sharply when the actual noise exceeds the value of $10^{-1}$. It is interesting to see that when we use the optimistic ${ }^{3}$ high probability estimates for $p_{A}, p_{U}$, we obtain almost always better performance than simply guessing the noise, or using the plain empirical estimate $\hat{\omega}$ directly. Furthermore, using the asymptotically optimal threshold (16), we observe a deterioration in the results. Thus, our approach results in a clearly dominating performance over other methods.

As mentioned in Sec. VI, the choice of the threshold by Baignères et al. [14] is only asymptotically optimal. Ours, while not optimal, gives a worst-case expected loss guarantee for any finite sample size. Thus, it has better performance when the asymptotic approximation is not sufficiently good, which occurs when both the number of rounds $n$ and the gap $\Delta$ are small.

\section{CONCLUSION}

We have performed the first, to our knowledge, expected loss analysis of additive error challenge-response authentication protocols under channel constraints. Such an analysis is necessary, because of the inherent cost of increasing the number of rounds $n$ and the need to trade off optimally the

\footnotetext{
${ }^{3}$ Experiments with pessimistic high probability estimates for the noise showed a significant increase in the number of rounds used, which resulted in a higher expected loss.
} 


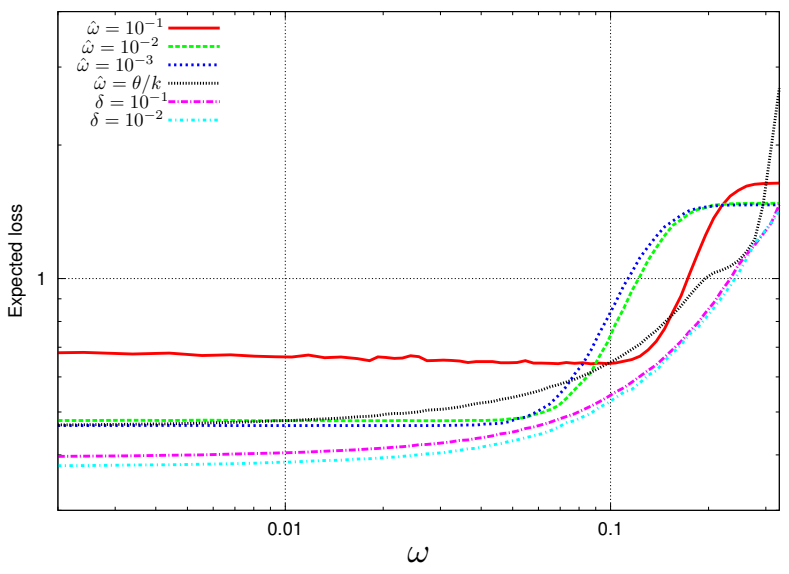

(a) Our threshold

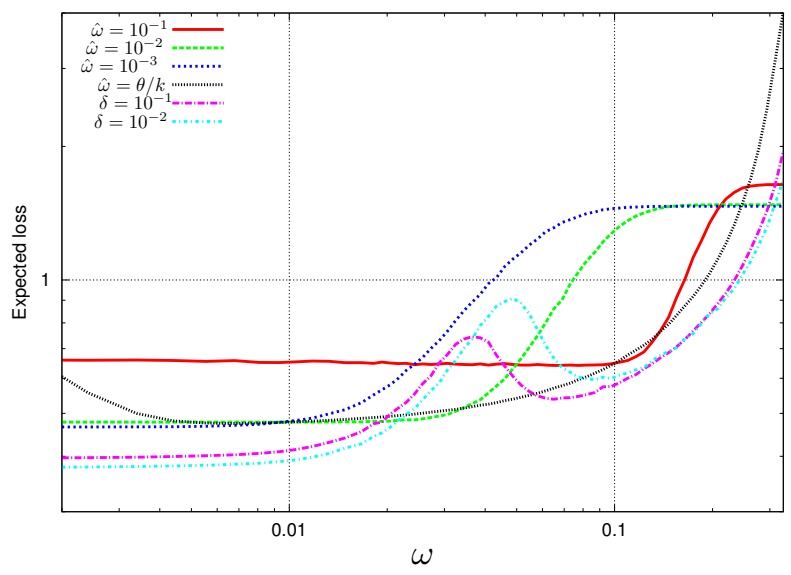

(b) Asymptotic threshold

Fig. 2. The worst-case expected loss as a function of noise. We plot the evolution of the loss as noise changes, for a number of different cases. Firstly, for the case where we arbitrarily assume a noise value $\hat{\omega} \in\left\{10^{-1}, 10^{-2}, 10^{-3}\right\}$. Secondly, for an empirically estimated $\hat{\omega}=\hat{k} / n$, and finally for $p_{A}, p_{U}$ calculated via equation (15) with $\delta \in\left\{10^{-1}, 10^{-2}\right\}$.

costs of incorrectly authenticating an illegitimate entity, or failing to authenticate a legitimate one, as well as the lack of an error-free channel. In order to achieve this, we made the assumption of bounds on the expected errors of a user and an attacker. In addition, we assumed that these are independent from errors in the previous rounds. ${ }^{4}$

The rapid-bit exchange phase considered in parts of this paper was introduced in [3] to compute an upper bound on the distance of the prover $\mathcal{P}$ from the verifier $\mathcal{V}$. This is composed of $n$ challenge-response rounds, used to calculate a round-trip time and thus place a bound on the distance. Subsequently, a broad range of distance bounding protocols were proposed, both for RFID [5], [7], [9]-[11], as well as other wireless devices [6], [19], [20].

Hancke and Kuhn [9] were the first to indicate that since the rapid-bit exchange phase is taking place in a noisy channel,

\footnotetext{
${ }^{4}$ Consequently, error probabilities drop exponentially fast, unlike the examples given in [18].
}

challenges and responses may be corrupted. Thus, a legitimate user may fail to get authenticated. Their protocol (henceforth HAKU), employed $n$ rounds and authenticated any prover who made a number of mistakes $\varepsilon$ less than an acceptance threshold $\tau$, so as to reduce the number of false rejections. Using the binomial distribution and an assumption on the error rates they give expressions for the false accept and false reject probability as a function of $n$ and $\tau$, but they provide no further analysis. Nevertheless, they indicate that the number of challenge-response rounds $n$ in the rapid bit exchange phase should be chosen according to the expected error rate. Kim et al. [11] extend this approach with the SwISS-KNIFE protocol by considering three types of errors. Finally [5], rather than using a threshold $\tau$, proposed a protocol (henceforth ECMAD) using an error correcting code (ECC). ECMAD, which extends the MAD protocol [19], uses only $k$ of the $n$ total rounds for the challenges and responses. The remaining $n-k$ rounds are used to transmit the $(n, k)$ ECC. This has the effect of achieving better security (in terms of false acceptance rates) with the same number of rounds $n$.

All these approaches use $n$ rounds in the noisy authentication phase. However, they do not define the optimal $n$. They simply state that the probability of authenticating a user becomes much higher than the probability of authenticating an attacker as $n$ increases. However, a large value of $n$ is incompatible with the requirements of many applications and devices (i.e. high value of $n$ leads to high overhead for resource-constrained devices). This can be modelled by assigning an explicit cost to every round, which should take into account the transmission energy, computation and time overhead. This cost has so far not been explicitly taken into consideration.

Another work that is closely related to ours is [21], which, given a required false acceptance and false rejection rate, provides a lower bound on the number of required rounds. This analysis is performed for both HAKU and ECMAD. However, it assumes that the number of rounds $n$ would be large enough for the binomial distribution of errors to be approximately normal. Our analysis is more general, since it uses finitesample bounds that hold for any bounded error function.

Recently, Baignères et al. [14] have given an analysis on the related topic of distinguishing between a real and a fake solver of challenge-response puzzles. More precisely, they study CAPTCHA-like protocols and provide a threshold which minimizes the probability of error in these protocols. The main differences between the analysis presented in this paper and [14] can be summarised below: (a) We perform an expected loss analysis rather than an error analysis. (b) Our bounds hold uniformly, while [14] uses an asymptotically optimal distinguisher. (c) We consider bounded errors rather than $\{0,1\}$ errors for each challenge-response. (d) We additionally propose a method to estimate channel noise. This is of course not applicable in the context of [14], due to the different setting.

A more general work on authentication under noisy conditions was presented in [22]. This provided tight information- 
theoretic upper and lower bounds on the attacker's success in impersonation and substitution attacks, proving that it decreased with noise. However, our analysis shows that, when one considers losses due to communication overhead and false rejections of users, the expected loss increases, which is a natural result.

In this paper, we perform a detailed expected loss analysis for a general class of additive-error authentication protocols in a noisy channel. The analysis is performed by assigning a loss $\ell_{B}$ to each round, and losses $\ell_{A}, \ell_{U}$ to false acceptance and false rejection respectively.

We show how a nearly-optimal threshold $\hat{\tau}_{n}^{*}$ for a given number of rounds $n$ can be chosen and give worst-case bounds on the expected loss for that choice. Thus, the bounds hold no matter if the party that attempts to get authenticated is either a legitimate user $U$ or an attacker $A$. This extends our previous work [7], which proposed a new distance bounding protocol (HiтомI) and only calculated a value for the threshold $\tau$, without providing any bounds.

We also show how a nearly-optimal number of rounds $\hat{n}^{*}$ can be chosen and give further bounds on the expected loss. The bounds hold for any bounded error function, and not only for $\{0,1\}$ errors. ${ }^{5}$ Furthermore, they are valid for any $n$, since they are based on probability inequalities for a finite number of samples. ${ }^{6}$ Thus, they are considerably more general to the bounds of [21].

Finally, we provided high-probability estimates for the current noise level in the channel by leveraging the coding performed in the initial and final phases of the protocol, which takes place in a coded channel. This enables us to significantly weaken assumptions on knowledge of the noise level in the channel and in turn, provide an authentication algorithm which has low expected loss with high probability. Experimentally, we obtain uniformly superior results to guessing or direct empirical noise estimates. Finally, we repeated those experiments with an asymptotically optimal threshold similar to that used by Baignères et al. [14]. Our results indicate a significant improvement through the use of a threshold with uniform, rather than asymptotic, guarantees. Consequently, it is our view that algorithms motivated by an asymptotic analysis should be avoided in the finite-sample regime of most challenge-response authentication protocols.

Our analysis is particularly significant for areas of communications where challenges and responses are costly and where there exists significant uncertainty about the correctness of any single response.

\section{ACKNOWLEDGEMENTS}

This work was partially supported by the Marie Curie IEF project "PPIDR: Privacy-Preserving Intrusion Detection and Response in Wireless Communications", grant number: 252323, the Marie Curie IEF Project "ESDEMUU: Efficient

\footnotetext{
${ }^{5}$ In all previous proposals, there is either an error at each round, or there is not.

${ }^{6}$ The analysis in [21] only holds for large $n$, so the approximation only holds asymptotically.
}

sequential decision making under uncertainty", Grant Number 237816 and the FP7 project "IM-CLeVeR - Intrinsically Motivated Cumulative Learning Versatile Robots", grant agreement No FP7-ICT-IP-231722.

\section{APPENDIX}

\section{A. Useful formulas}

If $X_{1}, \ldots, X_{n}$ are independent Bernoulli random variables with $X_{k} \in\{0,1\}$ and $\mathbb{P}\left(X_{k}=1\right)=\mu$ for all $k$, then

$$
\mathbb{P}\left(\sum_{k=1}^{n} X_{k} \geq u\right)=\sum_{k=0}^{u}\left(\begin{array}{l}
n \\
k
\end{array}\right) \mu^{k}(1-\mu)^{n-k} .
$$

This probability can be bounded via Hoeffding's inequality [23]:

Lemma 3 (Hoeffding). For independent random variables $X_{1}, \ldots, X_{n}$ such that $X_{i} \in\left[a_{i}, b_{i}\right]$, with $\mu_{i} \triangleq \mathbb{E} X_{i}$ and $t>0$ :

$$
\begin{aligned}
& \mathbb{P}\left(\sum_{i=1}^{n} X_{i} \geq \sum_{i=1}^{n} \mu_{i}+n t\right) \leq \exp \left(-\frac{2 n^{2} t^{2}}{\sum_{i=1}^{n}\left(b_{i}-a_{i}\right)^{2}}\right) \\
& \mathbb{P}\left(\sum_{i=1}^{n} X_{i} \leq \sum_{i=1}^{n} \mu_{i}-n t\right) \leq \exp \left(-\frac{2 n^{2} t^{2}}{\sum_{i=1}^{n}\left(b_{i}-a_{i}\right)^{2}}\right) .
\end{aligned}
$$

\section{B. On Bayesian hypothesis tests}

One way to obtain an asymptotically optimal threshold is to employ a Bayesian hypothesis test [15]. This requires defining a prior probability on the possible hypotheses. In our case, the hypothesis set is $H=\{A, U\}$, on which we define a prior probability $\pi$. For $\{0,1\}$ errors, the probability of observing $\varepsilon$ errors out of $n$ observations is given by $\mathbb{P}(\varepsilon \mid A)$ and $\mathbb{P}(\varepsilon \mid U)$ for the attacker and user respectively and it follows a binomial distribution with parameters $p_{A}, p_{U}$ respectively. Given an observed error $x$, the posterior probability of any hypothesis $h \in H$ is:

$$
\pi(h \mid \varepsilon=x)=\frac{\mathbb{P}(\varepsilon=x \mid h) \pi(h)}{\sum_{h^{\prime} \in H} \mathbb{P}\left(\varepsilon=x \mid h^{\prime}\right) \pi\left(h^{\prime}\right)} .
$$

We then define a decision set $G=\left\{g_{A}, g_{U}\right\}$, where $g_{A}$ means we decide that the prover is an attacker and $g_{U}$ means we decide that the prover is a user. Finally, we define a loss function $L: G \times H \rightarrow \mathbb{R}$, such that $L(g, h)$ is our loss when we decide $g$ and $h$ is the correct hypothesis. The expected loss of decision $g \in G$, under our prior and given $\varepsilon$ errors out of $n$ is:

$$
\mathbb{E}_{\pi}(L \mid \varepsilon, g)=\sum_{h \in H} L(g, h) \pi(h \mid \varepsilon),
$$

where $\mathbb{E}_{\pi}$ denotes expectation with respect to the prior $\pi$. Now define the decision function $q:\{0,1, \ldots, n\} \rightarrow G$ :

$$
q(\varepsilon) \triangleq \begin{cases}g_{U}, & \text { if } \mathbb{E}_{\pi}\left(L \mid \varepsilon, g_{U}\right) \leq \mathbb{E}_{\pi}\left(L \mid \varepsilon, g_{A}\right) \\ g_{A}, & \text { if } \mathbb{E}_{\pi}\left(L \mid \varepsilon, g_{U}\right)>\mathbb{E}_{\pi}\left(L \mid \varepsilon, g_{A}\right)\end{cases}
$$

This decision function minimises $\mathbb{E}_{\pi} L$ by construction (c.f. [15] ch. 8). The following remark is applicable in our case: 
Remark 1. Assume i.i.d errors with $\varepsilon_{i} \in\{0,1\}$, so that we can use a binomial probability for $\mathbb{P}(\varepsilon \mid h)$. Set the loss function $L$ to be $L\left(g_{U}, A\right)=\ell_{A}, L\left(g_{A}, U\right)=\ell_{U}$ and 0 otherwise. Then the decision function (19) becomes equivalent to:

$$
q(\varepsilon) \triangleq \begin{cases}g_{U}, & \text { if } \varepsilon<\tau_{b} \\ g_{A}, & \text { if } \varepsilon \geq \tau_{b}\end{cases}
$$

where

$$
\tau_{b} \triangleq \frac{n \ln \frac{1-p_{U}}{1-p_{A}}-\ln \left[\rho \frac{\pi(A)}{\pi(U)}\right]}{\ln \frac{1-p_{U}}{1-p_{A}}-\ln \frac{p_{U}}{p_{A}}}
$$

Proof: We start by calculating the expected loss for either decision. First:

$\mathbb{E}_{\pi}\left(L \mid \varepsilon, g_{A}\right)=\ell_{U} \pi(U \mid \varepsilon)=\frac{\ell_{U} \pi(U) \mathbb{P}(\varepsilon \mid U)}{\pi(A) \mathbb{P}(\varepsilon \mid A)+\pi(U) \mathbb{P}(\varepsilon \mid U)}$,

due to our choice of $L$ and $\pi$. Similarly,

$\mathbb{E}_{\pi}\left(L \mid \varepsilon, g_{U}\right)=\ell_{A} \pi(A \mid \varepsilon)=\frac{\ell_{A} \pi(A) \mathbb{P}(\varepsilon \mid A)}{\pi(A) \mathbb{P}(\varepsilon \mid A)+\pi(U) \mathbb{P}(\varepsilon \mid U)}$

Combining the above expressions, the decision function (19) can then be written so that we make decision $g_{U}$ if and only if:

$$
\ell_{A} \pi(A) \mathbb{P}(\varepsilon \mid A) \leq \ell_{U} \pi(U) \mathbb{P}(\varepsilon \mid U) .
$$

Finally, replacing (17) with means $p_{A}, p_{U}$ respectively and taking logarithms we obtain:

$$
\ln [\rho \pi(A) / \pi(U)]+\varepsilon \ln \frac{p_{A}}{p_{U}} \leq(n-\varepsilon) \ln \frac{1-p_{U}}{1-p_{A}},
$$

as a condition for deciding $g_{U}$. With some elementary manipulations, we arrive at the required result.

Given the conditions of the previous remark, it is easy to see (c.f. [15] ch. 8) that the decision function $q$ minimises the Bayes risk:

$$
\mathbb{E}_{\pi}(L \mid q)=\pi(A) \mathbb{P}\left(\varepsilon<\tau_{b} \mid A\right) \ell_{U}+\pi(U) \mathbb{P}\left(\varepsilon \geq \tau_{b} \mid U\right) \ell_{A} .
$$

Furthermore, for $\pi(A)=\pi(U)=1 / 2$, we obtain (16). In addition, this choice also minimises an upper bound on the worst-case expected loss since:

$$
\max _{h \in H} \mathbb{E}(L \mid h, q) \leq \sum_{h \in H} \mathbb{E}(L \mid h, q)=2 \mathbb{E}_{\pi}(L \mid q) .
$$

for uniform $\pi$.

Finally, the asymptotic optimality of Bayesian testing generally follows from Bayesian consistency (c.f. [15] ch. 10). More specifically, [17] has proved the asymptotic optimality of Bayes solutions for hypothesis testing of the type examined here.

\section{REFERENCES}

[1] W. Stallings, Cryptography and Network Security. Prentice Hall, 2006.

[2] D. R. Stinson, Cryptography: theory and practice. Chapman \& Hall, 2006.

[3] S. Brands and D. Chaum, "Distance-bounding protocols," in Proc. EUROCRYPT'93, ser. LNCS, vol. 765, 1994, pp. 344-359.

[4] L. Bussard and W. Bagga, "Distance-bounding proof of knowledge to avoid real-time attacks," in Security and Privacy in the Age of Ubiquitous Computing, vol. 181. Springer Boston, 2005, pp. 223-238.

[5] D. Singelée and B. Preneel, "Distance bounding in noisy environments," in Security and Privacy in Ad-hoc and Sensor Networks - ESAS 2007, ser. LNCS, vol. 4572. Springer, 2007, pp. 101-115.

[6] N. O. Tippenhauer and S. Capkun, "ID-Based Secure Distance Bounding and Localization," in Computer Security - ESORICS 2009, ser. LNCS, vol. 5789. Springer Berlin / Heidelberg, 2010, pp. 621-636.

[7] P. Peris-Lopez, J. C. Hernandez-Castro, C. Dimitrakakis, A. Mitrokotsa, and J. M. E. Tapiador, "Shedding light on RFID distance bounding protocols and terrorist fraud attacks," arXiv:0906.4618v2, 2010.

[8] C. Kim and G. Avoine. (2009) RFID distance bounding protocol with mixed challenges to prevent relay attacks. Cryptology ePrint Archive, Report 2009/310.

[9] G. Hancke and M. Kuhn, "An RFID distance bounding protocol," in Proc. SECURECOMM'05, Sept. 2005, pp. 67-73.

[10] J. Reid, J. M. G. Nieto, T. Tang, and B. Senadji, "Detecting relay attacks with timing-based protocols," in Proc. ASIACCS'07. ACM, 2007, pp. 204-213.

[11] C. Kim, G. Avoine, F. Koeune, F. X. Standaert, and O. Pereira, "The Swiss-knife RFID distance bounding protocol," in Proceedings of ICISC '08, ser. LNCS. Springer-Verlag, Dec. 2008.

[12] C. Dimitrakakis, A. Mitrokotsa, and S. Vaudenay, "Expected loss analysis of thresholded authentication protocols in noisy conditions," arXiv, Tech. Rep. 1009.0278, 2011.

[13] Y. Desmedt, "Major security problems with the "unforgeable" (Feige)Fiat-Shamir proofs of identity and how to overcome them," in Proc. SecuriCom '88, Mar. 1988, pp. 147-159.

[14] T. Baignères, P. Sepehrdad, and S. Vaudenay, "Distinguishing distributions using Chernoff information," in ProvSec 2010. Springer-Verlag, 2010.

[15] M. H. DeGroot, Optimal Statistical Decisions. John Wiley \& Sons, 1970.

[16] H. Chernoff, "Sequential design of experiments," Annals of Mathematical Statistics, vol. 30, no. 3, pp. 755-770, 1959.

[17] _ _A measure of asymptotic efficiency for tests of a hypothesis based on the sum of observations," Annals of Mathematical Statistics, vol. 23, no. 4, pp. 493-507, 1952.

[18] M. Bellare, R. Impagliazzo, and M. Naor, "Does parallel repetition lower the error in computationally sound protocols?" in focs. Published by the IEEE Computer Society, 1997, p. 374.

[19] S. Čapkun, L. Buttyán, and J. Hubaux, "Sector: Secure tracking of node encounters in multi-hop wireless networks," in ACM SASN, October 2003, pp. 21-32.

[20] S. Čapkun and J. Hubaux, "Secure positioning in wireless networks," IEEE Journal on Selected Areas in Communications, vol. 24, pp. 221232, 2006.

[21] D. Singelée, "Study and design of a security architecture for wireless personal area networks," Ph.D. dissertation, Katholieke Universiteit Leuven, December 2008.

[22] L. Lai, H. E. Gamal, and H. V. Poor, "Authentication over noisy channels," IEEE Transactions on Information Theory, vol. 55, no. 2, pp. 906-916, February 2009.

[23] W. Hoeffding, "Probability inequalities for sums of bounded random variables," Journal of the American Statistical Association, vol. 58, no. 301, pp. 13-30, March 1963. 